\title{
Bottlenecks in Passenger Throughout at The Airport
}

\author{
Chunyan Ao \\ ${ }^{1}$ School of North China Electric Power University, Baoding 071003, China \\ aochunyan7@163.com
}

Keywords: simulation model, bottlenecks, flow, time consumption.

\begin{abstract}
In this paper, The Generalized Stochastic Petri Net simulation model is established to analyze the flow of passengers, bottlenecks, potential modifications, cultural differences' influence, and several recommendations are given to security managers.First, the current security checkpoint process in US airport is subdivided into 9 steps which are more detailed. The Generalized Stochastic Petri Net is built to analyze the flow of passengers considering the Synchronization and Concurrency of passenger's security check process, with time parameter contained also. The Average Token which illustrates the average time consumption in the nine check steps is shown in Table 1. The steps in which the Token value is big are seemed as the critical step. The Monte Carlo method is adapted then to analyze the bottlenecks in check process. The simulation result (in Table 4) shows the time cost in each critical step, and the bottlenecks are found lying in three aspects: Open-package inspection, Body screening and Retrieving luggage.
\end{abstract}

\section{Introduction}

With the acceleration of economic globalization, more and more people like choosing plane as their common transportation because of its high speed and wide range of region they can get. However, it is annoying to wait in the line at the airport for security check for a long time, sometimes may missing the flight. This phenomenon is more and more common recently. For example, long lines reported at Chicago Midway Airport's TSA checkpoint on July 13, 2016. It is said that The TSA estimated a wait time of up to two-hours at Midway Airport's main security checkpoint ${ }^{[1]}$. So, it is urgent for our team to do some research then make an analysis finally put forward some significant proposal to solve these problems. Where problem areas bottlenecks exist in the current process?A full analysis is discussed in this paper.

\section{Analyzing the flow of passengers.}

The section Using the data in O’Hare airport to build the Petri network, and the analysis is made then to get its property. Statistics in each step of the security check process is analyzed then.

Table 1 The data of security-check service in O’Hare airport

\begin{tabular}{lllll}
\hline Action & $\begin{array}{l}\text { Time } \\
\text { cost/s }\end{array}$ & $\mathrm{Ti}$ & Symbol & $\begin{array}{l}\text { Symbol } \\
\text { value }\end{array}$ \\
\hline ID check & 15 & $t_{1}$ & $\lambda_{1}$ & 4 \\
Queue & 10 & $t_{2}$ & $\lambda_{2}$ & 6 \\
X-ray scan & 5 & $t_{3}$ & $\lambda_{3}$ & 12 \\
FS & ---- & $t_{4}$ & $\mathrm{a}_{1}$ & 0.1 \\
OOBA & 100 & $t_{5}$ & $\lambda_{5}$ & 0.6 \\
NS & ---- & $t_{6}$ & $1-\mathrm{a}_{1}$ & 0.9 \\
PSC & 2.5 & $t_{7}$ & $\lambda_{7}$ & 24 \\
NSWarn & ---- & $t_{8}$ & $\mathrm{a}_{2}$ & 0.01 \\
SWarn & ---- & $t_{9}$ & $1-\mathrm{a}_{2}$ & 0.99 \\
PE & 30 & $t_{10}$ & $\lambda_{10}$ & 2 \\
TB & 60 & $t_{11}$ & $\lambda_{11}$ & 1 \\
\hline
\end{tabular}

Regarding the security check procedure as a system, we find the system having the characteristic of synchronicity and concurrency. Also, based on the actual situation of security-check system ${ }^{[2]}$, we 
introduce the time parameter to analyze the activity of the model changing over time. Thus, the Generalized Stochastic Petri Net, equals to GSPN is used to model the security-check procedure [1].

The petri-network model is simple and it have one ID check point, one detector gate, one X-ray machine and one inspector, as is shown in Figure below:

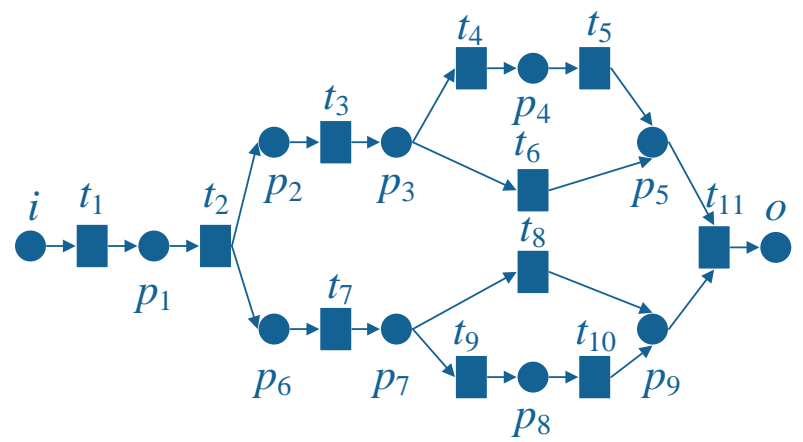

Fig. 1 The Petri net for tourist security check process

t1 is ID and boarding check; $t 2$ is Passenger waiting for security check; t3 is Luggage going through the $\mathrm{X}$-ray check; $\mathrm{t} 4$ is Luggage being suspected; $\mathrm{t} 5$ is Luggage being re-inspecting ; 6 is No hazards; t7 is Passenger passing the inspection door; 18 is No alarming; t9 is Alarming;t10 is Body screening; $\mathrm{t} 11$ is Retrieve for baggage and exit.

Based on the Petri network mentioned above, taking the given time data and probability distribution to instead the corresponding time vicissitude and instantaneous change in the map. The time vicissitude $t$ and the average instant rate $\lambda$ can be added between the inputs $i$ and the output $o$, and then the GSPN is get. The GSPN is equals to a Markov Chain (MC) with the continuous time. The state probability and be get through the MC method, and the signed probability density, the average signed number in the library, the using rate of the transition can also get.

(1) Building GSPN model for security-check system. The service time obeys the Negative Exponential Distribution, so does the delay time. The initiation probability should also be assigned for each transform. The GSPN model for the passenger security-check system is shown as below:

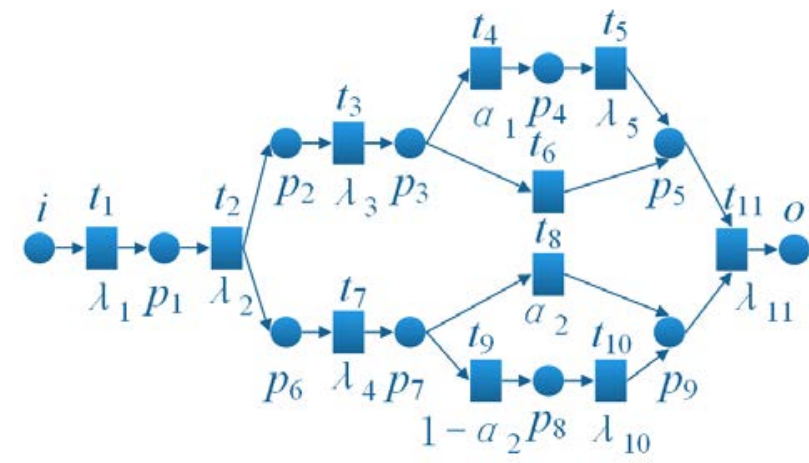

Fig. 2 The GSPN model for tourist security check system

$\mathrm{T}_{\mathrm{a}}$ is The collection of the time change $\left(\mathrm{T}_{\mathrm{a}}=\{\mathrm{t} 1, \mathrm{t} 2, \ldots, \mathrm{t} 11\}\right) ; \lambda \mathrm{a}$ is The trigger rate in accordance with time change $(\lambda \mathrm{a}=\{\lambda 1, \lambda 2, \ldots, \lambda 11\})$; Tb is The collection of the moment change $(\mathrm{Tb}=$ $\{t 4, t 6, t 8, t 9\})$.If the lace (p3) is in the condition of Token, the moment change : $t 4$ and $t 6$ can be operated. $\alpha 1$ is the probability of the change on $t 4 ; 1-\alpha 1$ is the probability of the change on t6; $\alpha 2$ is the probability of the change on t8; $1-\alpha 2$ is the probability of the change on $t 9$.

(2) Using the built GSPN network, we can get the reach ability graph. After processing, we can get the MC network which has the same structure with the GSPN model. Using the MC network, we can solve the probability in the stable stage, and the time in the security- check procedure is calculated. The improvement using compared with the previous conditions can get, including improve the passenger flow and increase the variance in wait time.

The density matrix is built for the MC network:

The real state: ( M1， M2, M4， M6， M9, M11 ,M12, M15， M16， M17) 
The disappeared state: ( M3, M5, M7, M8, M10, M13, M14) can be ignored and we can simplify the GSPN thus the MC network can get as follows:

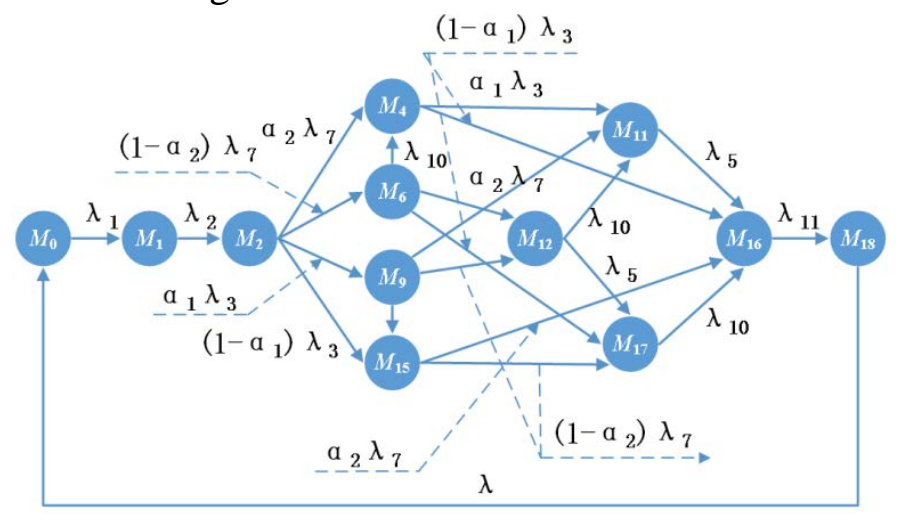

Fig. 3 The Isomorphism Markov Chain

(3) Solving the stable probability for MC: using the density matrix $\mathrm{Q}$ and the formulation $X Q=0$, we can get the probability distribution in the stable state.

(4) Analyzing the performance improvement for the security check system. Using the probability distribution in the stable state, we can get the average Token value in each step, thus we can analyze the performance index.

Table 2 Average Tolkien in each Library

\begin{tabular}{llll}
\hline Library & Toa & Transmit & $R_{t}$ \\
\hline$i$ & 0.10105 & $t_{1}$ & 0.10105 \\
$P_{1}$ & 0.07924 & $t_{3}$ & 0.07924 \\
$P_{2}$ & 0.01836 & $t_{4}$ & 0.01836 \\
$P_{3}$ & - & $t_{5}$ & 0.08353 \\
$P_{4}$ & 0.03245 & $t_{8}$ & 0.01245 \\
$P_{5}$ & 0.47624 & $t_{10}$ & 0.17624 \\
$P_{6}$ & 0.01839 & $t_{11}$ & 0.30839 \\
$P_{7}$ & - & & \\
$P_{8}$ & 0.07625 & & \\
$P_{9}$ & 0.13271 & & \\
$o$ & 0.42536 & & \\
\hline
\end{tabular}

\section{Identifying bottlenecks in check process.}

Based on Monte Carlo Method, we make an analysis for the Bottlenecks in the zones. First, based on the time record for different steps of process the table provided and the other data we have searched, the critical steps of the security check are affirmed: Start $\rightarrow A \rightarrow B \rightarrow G \rightarrow H \rightarrow I \rightarrow J \rightarrow K \rightarrow$ End. Then, by MCM, we make an analysis for the critical steps.

The time of whole process and time of single step are considered. Every step of the process is discrete and random. Thus we define random factors influencing the time of every step as the simulation variables.

$$
x_{i} \in\left\{x_{1}, x_{2}, \cdots, x_{m}\right\}
$$

Service time of each step gains random values in accordance with the negative exponential distribution. Thus, the total time of the process is as follow:

$T_{k}=T_{k}\left(x_{1}\right)+T_{k}\left(x_{2}\right)+\cdots+T_{k}\left(x_{m}\right)$

Then, we complete $\mathrm{N}$ simulation experiments (the processes is shown in Figure3.) and get the maximum and minimum

$$
\begin{array}{cl}
T_{\text {max }}=\max \left\{T_{k}\right\} & k \in\{1,2, \cdots, N\} \\
T_{\text {min }}=\min \left\{T_{k}\right\} & k \in\{1,2, \cdots, N\}
\end{array}
$$


When the simulation process attends to reach the steady state, the expected values of whole process and single step after $\mathrm{N}$ experiments is gained. The process is shown in Figure 4 below.

The expected value of whole process: $E(T)$

The expected value of single process: $E\left(T\left(x_{i}\right)\right)$

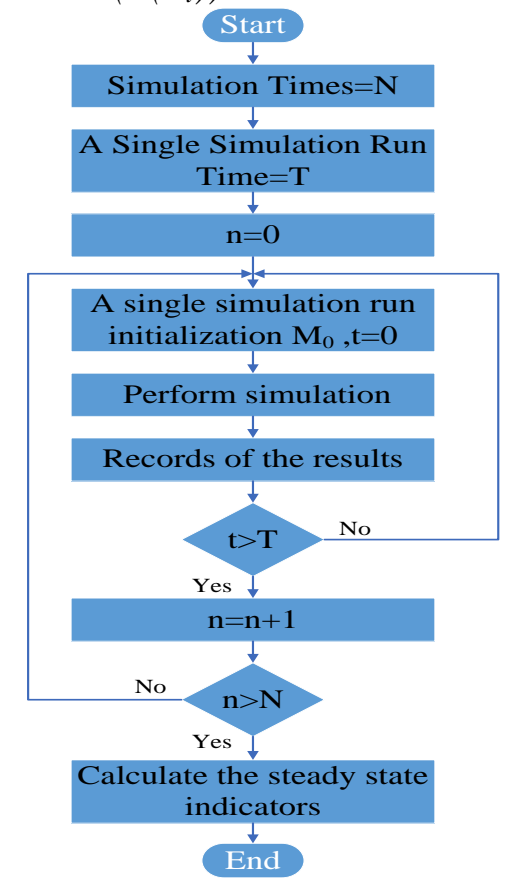

Fig. 4 The flow chart of Monte Carlo method

Then, we get two significant metrics: time difference between two steps and proportion of the single step taking part in the time of whole process.

Time difference:

$\Delta T\left(x_{i}\right)=T_{\max }\left(x_{i}\right)-T_{\min }\left(x_{i}\right)$

Proportion:

$$
p=\frac{E\left(T\left(x_{i}\right)\right)}{E(T)}
$$

If the i step is big, it indicates that the step is random and has a significantly influence on the whole security check process, which increases the check time for passengers.

If p of one step taking up the whole process is big, it indicates that this step is a main step for the whole process and has a possibility of becoming the bottlenecks of the security check.

After 250 iterations being completed, the results are shown in Table 3.

Table 3 The value of time difference and proportion of the critical steps

\begin{tabular}{|lllll|}
\hline Step & Tmax & Tmin & & P \\
\hline A & 15 & 5.4 & 9.6 & $6.2 \%$ \\
\hline B & 16.8 & 5.3 & 11.5 & $7.4 \%$ \\
\hline C & 30.8 & 12.9 & 17.9 & $11.6 \%$ \\
\hline D & 31.2 & 15.3 & 15.9 & $10.3 \%$ \\
\hline G & 35.6 & 5.6 & 30 & $19.4 \%$ \\
\hline F & ---- & ---- & ---- & ---- \\
\hline H & 25.6 & 0 & 25.6 & $16.5 \%$ \\
\hline I & 25.3 & 6.3 & 19 & $12.3 \%$ \\
\hline K & 30.6 & 5.7 & 24.9 & $16.1 \%$ \\
\hline
\end{tabular}

Where:

A------ Checking passengers' identification and ticket, boarding pass

B------ Instructing and assisting passengers to put the luggage on the conveyor belt 
C------Waiting for the movement of the conveyor belt

D------The inspectors monitor X-ray tester and complete discrimination

E------ Judge conditions: passenger baggage is suspected of illegal items. 95\% of the passengers need to open baggage for check $5 \%$ of passengers don't need

F------ Enter zone D for the re-inspection G------Instructing passengers to put keys, wallet and other metal objects into the tray

H------ Waiting the passengers in front completing personal check

I------ Passengers put off shoes, belts, jackets, metal objects accept personal check gate

J------ Judge conditions: whether the passenger passes the millimeter wave scanner or metal detector

K------passengers retrieve luggage

The results in Table1 illustrates that bottlenecks of the security check are:

1) putting keys, wallet and other metal objects into the tray;

2) waiting the passengers in front completing personal check;

3) retrieving luggage.

\section{Conclusion}

The three steps have the biggest time portion: 30s, 25.6s and 24.9s respectively, which indicates the variance causing by low efficiency of both passengers and staffing. From the proportion of the three steps taking the whole process,there are three main bottlenecks: putting keys, wallet and other metal objects into the tray; waiting the passengers in front completing personal check and retrieving luggage which respectively exist in Document Check Zone, Baggage and Body Screening zone and Collect Items and Exit Zone.

\section{References}

[1]. Dijknm,Erikvds. Check-in computation and optimization by simulation and IP in combination [J]. European Journal of Operational Research, 2006,171:1152-1168.

[2]. JiangXinxin, ZhouHang, CaiBingqing. Optimization R esearch on Process and Layout of Terminal Security Check [J]. Southern airlines computing technology,2015,(03):25-29. 